\title{
Análise morfológica do arco superior de portadores de fissura labiopalatal submetidos a diferentes protocolos de expansão rápida maxilar: avaliação das alterações maxilares
}

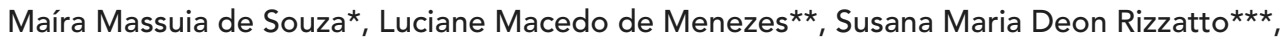

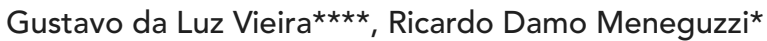

\section{Resumo}

Introdução: em portadores de malformações labiopalatais, a boa conformação do arco superior constitui um dos principais requisitos para a obtenção de uma oclusão adequada e para o aperfeiçoamento estético e funcional. Para tanto, a expansão ortopédica da maxila é frequentemente necessária, uma vez que é alta a incidência de colapso dos segmentos. Objetivos: analisar, por meio de modelos de gesso, as dimensões do arco superior de 15 portadores de fissura transforame incisivo unilateral, com deficiências transversal e anteroposterior da maxila, submetidos à expansão com dois diferentes protocolos de ativação. Métodos: um grupo foi submetido à Expansão Rápida da Maxila (ERM) com protocolo de ativação convencional (Grupo 1), enquanto no outro grupo foi utilizado o protocolo de ativação preconizado por Liou e Tsai em 2005, com expansões e contrações alternadas (Grupo 2), sendo ambos submetidos posteriormente à protração maxilar com máscara de tração reversa. Os modelos foram obtidos em dois momentos: antes da cimentação do disjuntor do tipo Haas modificado (T1) e após 24 semanas de tratamento (T2). As distâncias transversais entre os primeiros pré-molares superiores (região anterior) e a distância entre os primeiros molares superiores (região posterior) foram registradas com um paquímetro digital. Os dados obtidos foram submetidos ao teste $t$ de Student. Resultados e Conclusões: os dois protocolos de ativação mostraram-se igualmente efetivos na expansão maxilar, não havendo diferença entre os grupos em relação ao aumento transversal obtido.

Palavras-chave: Expansão maxilar. Distância interpré-molares. Distância intermolares. Fissura labiopalatal.

\section{INTRODUÇÃO}

O fissurado transforame incisivo unilateral apresenta um crescimento maxilar diferente do indivíduo não-fissurado, em todos os sentidos, sendo que, ao nascimento, as dimensões anteroposterior e transversal da maxila se encontram aumentadas $^{7}$. Por volta dos três anos, as relações anteroposteriores e vertical apresentam-se

* Mestres em Ortodontia e Ortopedia Facial pela Faculdade de Odontologia da PUCRS

** Doutora e mestre em Ortodontia pela UFRJ. Professora de Ortodontia na Faculdade de Odontologia da PUCRS.

*** Mestre em Ortodontia e Ortopedia Facial pela PUCRS. Professora de Ortodontia na Faculdade de Odontologia da PUCRS **** Mestre em Ortodontia e Ortopedia Facial pela PUCRS. 
próximas do normal e, a partir dessa idade até os seis anos, passa a existir uma tendência à retrusão maxilar ${ }^{24}$. Uma característica desses pacientes é o perfil côncavo, resultado do retrognatismo da maxila devido à falta de crescimento anteroposterior da mesma ${ }^{24}$, levando ao prognatismo mandibular relativo ou à pseudo-Classe III. Dessa forma, há alta incidência de mordida cruzada anterior, evidente na transição entre as dentaduras decídua e mista. O desenvolvimento de mordida aberta também é comum, devido à deficiência no crescimento vertical da maxila ${ }^{8}$.

No estudo de Athanasiou et $a{ }^{4}{ }^{4}$, os autores compararam as dimensões dos arcos dentários de 72 crianças portadoras de fissura transforame incisivo unilateral operadas com as de crianças sem fissura. As dimensões foram estudadas em modelos de gesso durante as épocas de dentadura decídua completa (3-4 anos de idade), dentadura mista (8-9 anos de idade) e dentadura permanente (12 anos). Os autores concluíram que todas as larguras maxilares transversais e anteroposteriores foram significativamente menores nos pacientes com fissura do que naqueles sem fissura, em todas as idades, exceto a largura intermolares aos 12 anos.

Após a cirurgia do lábio, realizada nos primeiros meses de vida, esses pacientes frequentemente apresentam subdesenvolvimento maxilar, envolvendo as dimensões sagital, vertical e transver$\mathrm{sal}^{12,26}$, principalmente devido à presença de cintas musculares labiais firmes e tensas, bem como de tecidos cicatriciais no palato que influenciam negativamente o crescimento ${ }^{6,17}$, além de acarretar grande prejuízo estético e funcional ${ }^{17}$.

As alterações faciais mais frequentes em quase todos os tipos de fenda, provavelmente resultantes dos atos cirúrgicos, são: retrusão da face média, distorção das estruturas alveolares e alterações na postura da mandíbula ${ }^{21}$. A Ortodontia é indispensável no tratamento das sequelas maxilares provocadas pelas cirurgias, cuja gravidade está diretamente relacionada ao grau de traumatismo provocado pelas mesmas ${ }^{14}$.
A maioria dos pacientes submetidos à cirurgia sem assistência ortodôntica subsequente chega à vida adulta com uma situação alterada dos seguimentos maxilares, ocasionando o desenvolvimento de más oclusões de difícil tratamento e prognóstico ruim. As mordidas cruzadas posteriores são frequentes, apresentando vários graus de severidade, envolvendo desde um único dente até o arco todo, com tendência ao agravamento durante a dentadura permanente ${ }^{16}$. A retrusão da maxila associada à mordida cruzada anterior e lateral é um achado muito comum em pacientes portadores de fissura, apesar de todo o acompanhamento e cuidados despendidos durante o tratamento ${ }^{18}$.

\section{Tratamento da mordida cruzada em fissurados}

As intervenções ortodônticas interceptativas em pacientes sob programas de reabilitação devem ser executadas após os seis anos de idade ${ }^{2}$, pois nessa fase os mesmos apresentam as distâncias transversais anteriores (intercaninos) quase sempre diminuídas, acarretando o estreitamento na região anterior da maxila - prejudicial ao posicionamento da língua e, consecutivamente, ao tratamento fonoaudiológico executado nessa época. Além disso, é nessa fase que o crescimento da maxila começa a se manifestar com mais intensidade e os pacientes se mostram mais conscientes, exigentes e colaboradores com o tratamento ${ }^{1}$.

Devido às alterações no crescimento da maxila, os procedimentos mais comumente realizados durante a dentadura mista são: expansão maxilar para a correção da dimensão transversal reduzida; alinhamento e protrusão de incisivos, solucionando as rotações, apinhamentos e mordidas cruzadas anteriores; e protração maxilar para corrigir a retrusão da maxila ${ }^{16}$. O estabelecimento de uma relação intermaxilar normal pela simples expansão promove condições para a reabilitação no futuro, uma vez que, após a resolução da mordida cruzada, os maxilares têm condições de crescer normalmente, favorecendo a irrupção dos dentes 
permanentes em posições mais adequadas ${ }^{27}$.

Apesar de promover melhora na relação transversal dos arcos, a expansão do arco superior não reflete nenhum resultado no perfil de pacientes com fissura transforame unilateral ${ }^{26}$. Dessa forma, a protracão maxilar é recomendada no tratamento da retrusão da face média, sendo instituída precocemente para tirar proveito do fato das suturas circum-maxilares estarem em crescimento ativo.

A instalação de aparelho fixo com parafuso expansor promove a expansão segmentada nos portadores de fissuras completas por meio do alargamento da fissura, sendo o efeito ortopédico resultante do posicionamento lateral dos segmentos palatinos $^{16,23}$. No indivíduo fissurado, a expansão não é seguida de formação óssea ao nível da sutura palatina mediana, devendo-se manter uma contenção após a remoção do aparelho até que um enxerto ósseo seja realizado na região ${ }^{13}$.

Apesar da ausência da sutura mediana na maxila dos fissurados, a expansão enfrenta restrições semelhantes às impostas nos pacientes sem fissura, pois as outras suturas da maxila oferecem uma grande resistência, sendo indicado o uso de aparelhos fixos dentomucossuportados ${ }^{7,11}$.

O aparelho expansor do tipo Haas é o mais utilizado para a expansão rápida da maxila e segue o mesmo protocolo de ativação utilizado para pacientes não-fissurados ${ }^{13}$. Ele pode ser associado à protração, funcionando como um esplinte para os dentes superiores, transmitindo a força a toda a maxila ${ }^{28}$.

Liou e Tsai ${ }^{15}$, em 2005, propuseram um novo protocolo de ativações para a ERM, utilizando um expansor com duas dobradiças para expandir e girar os processos maxilares com centro de rotação na região da tuberosidade. $\mathrm{O}$ protocolo consiste em expansões e contrações alternadas semanalmente, com ativações de uma volta completa por dia, durante sete a nove semanas. Segundo os autores, os procedimentos alternados promovem a desarticulação das suturas circum-maxilares sem expandir excessivamente a maxila, sendo indicados principalmente para pacientes que não necessitam de um grande ganho transversal. Em seu estudo, dez pacientes portadores de fissura labiopalatal foram submetidos a esse protocolo durante nove semanas, sendo comparados a um grupo de 16 pacientes, também fissurados, onde foi utilizado o protocolo convencional de $\mathrm{Haas}^{9}$ por uma semana. No grupo submetido ao novo protocolo, foi verificado maior deslocamento anterior da maxila.

Essa pesquisa objetivou analisar quantitativamente, por meio de pontos situados nas estruturas dentárias, os resultados obtidos com a expansão maxilar por meio de dois protocolos de ativação.

\section{MATERIAL E MÉTODOS \\ Caracterização da amostra}

$\mathrm{Na}$ presente pesquisa, 20 pacientes com fissura transforame incisivo unilateral, com atresia maxilar (transversal e anteroposterior) e idades entre 7 e 14 anos, que realizaram as cirurgias primárias reabilitadoras (queiloplastia e palatoplastia), foram inicialmente selecionados para o procedimento de expansão e protração maxilar.

A seleção da amostra foi baseada em escolha aleatória de indivíduos brasileiros, sendo todos leucodermas, dos gêneros masculino e feminino, e que procuraram tratamento ortodôntico no ambulatório do Centro de Reabilitação de Fissura Labiopalatina (CERLAP) da Faculdade de Odontologia da Pontifícia Universidade Católica do Rio Grande do Sul (PUCRS) no período compreendido entre os anos de 2005 e 2006.

Devido às ausências de elementos dentários essenciais para a realização das medidas utilizadas, apenas 15 pacientes foram admitidos, sendo a amostra final composta por sete indivíduos do gênero masculino e oito do gênero feminino, divididos em dois grupos.

Um grupo, composto por sete indivíduos, foi submetido à Expansão Rápida da Maxila (ERM) com protocolo de ativação convencional (Grupo 1), enquanto o outro grupo, com oito integrantes, 
foi submetido à ERM com protocolo de ativação preconizado por Liou e Tsai ${ }^{15}$, com expansões e contrações alternadas (Grupo 2), sendo ambos submetidos posteriormente à protração maxilar com máscara de tração reversa.

\section{Protocolo de tratamento}

A ERM foi realizada com o aparelho disjuntor tipo Haas modificado - composto por anéis nos primeiros molares permanentes e primeiros molares decíduos (ou primeiros pré-molares) - confeccionado com fio de aço inoxidável de $1,0 \mathrm{~mm}$ de diâmetro (Morelli ${ }^{\circledR}$, ref. 55.01.100, Sorocaba, SP, Brasil) e soldado aos anéis por vestibular e palatino. $\mathrm{Na}$ mesial dos anéis dos primeiros prémolares foram confeccionados ganchos para protração maxilar. O parafuso expansor $\left(\right.$ Morelli $^{\circledR}$, ref. 65.05 .008 ) utilizado possuía passe de $0,8 \mathrm{~mm}$ e abertura de $11 \mathrm{~mm}$.

O protocolo de tratamento para o Grupo 1 foi de uma semana de ERM, seguida de 23 semanas de protração maxilar, perfazendo um total de 24 semanas (aproximadamente 6 meses) de expansão e protração maxilar. A ativação do parafuso iniciou-se no mesmo dia da cimentação, com $0,8 \mathrm{~mm}$ por dia, durante uma semana. A primeira ativação foi de $4 / 4$ de volta (1 volta completa) e, nos dias subsequentes, a ativação foi de $2 / 4$ de volta pela manhã e $2 / 4$ de volta à noite.

Após uma semana da fase ativa da ERM, o parafuso expansor foi estabilizado com fio de amarrilho de 0,30mm de diâmetro (Morelli ${ }^{\circledR}$, ref. 55.01.225). Foi iniciada, então, a protração da maxila com máscara facial tipo Petit (GAC International Inc., ref. 17.100.20, NY, EUA) com elásticos de protração (Morelli ${ }^{\circledR}$, ref. 60.01.212) adaptados aos ganchos localizados na distal dos caninos superiores, sendo trocados semanalmente. A tensão nos elásticos foi de $500 \mathrm{~g}$ de cada lado.

Os pacientes foram instruídos a usar a máscara facial durante um período de 24 horas por dia, ou o máximo possível.

Para o Grupo 2, o protocolo de ativação foi de sete semanas alternadas de expansões e contrações rápidas da maxila - iniciando-se pela expansão -, seguidas de 17 semanas de protração maxilar, completando o período de 24 semanas de tratamento. A sequência foi de seis sessões semanais alternadas de expansões e contrações da maxila, seguidas por uma semana de expansão final, com ativações de $0,8 \mathrm{~mm}$ por dia (uma volta completa do parafuso), conforme descrito por Liou e Tsai ${ }^{15}$.

A máscara facial foi instalada após as sete semanas de expansões e contrações alternadas da maxila e seguiu-se o mesmo protocolo de tratamento do Grupo 1.

\section{Documentação}

Os modelos de gesso foram obtidos antes da cimentação do disjuntor do tipo Haas modificado (T1) e após as 24 semanas de tratamento (T2) em ambos os grupos. As moldagens foram realizadas com alginato manipulado, de acordo com as especificações do fabricante, e os moldes vazados com gesso tipo-pedra especial, em razão de sua maior reprodução de detalhes.

\section{Medidas utilizadas}

Após a obtenção dos modelos de gesso, as distâncias transversais foram registradas com um paquímetro digital (Mitutoyo Sul Americana Ltda, Suzano, SP) tanto na região anterior como na posterior do arco superior (Fig. 1), sendo analisadas as seguintes dimensões:

- Distância interpré-molares superiores: correspondente à região anterior do arco, sendo considerada a distância entres as pontas das cúspides palatinas dos primeiros pré-molares (14 e 24). Quando esses estavam ausentes, foi considerada a distância entre as pontas das cúspides mesiopalatinas dos primeiros molares decíduos (54 e 64).

- Distância intermolares superiores: correspondente à região posterior do arco, sendo considerada a distância entre as pontas das cúspides mesiopalatinas dos primeiros molares superiores permanentes (16 e 26). 

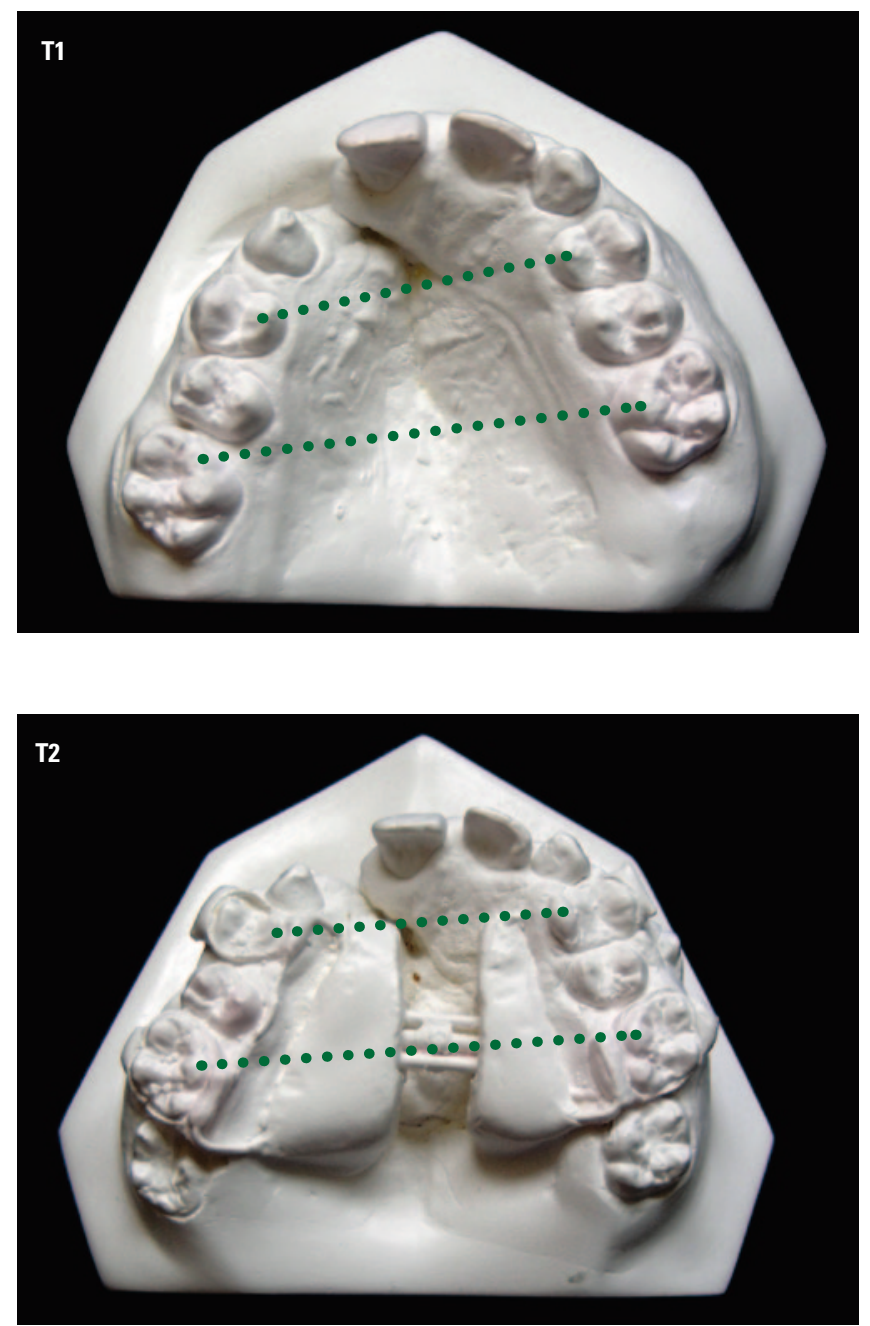

FIGURA 1 - Modelos ortodônticos antes (T1) e após a expansão rápida da maxila (T2), e distâncias transversais utilizadas: distância interpré-molares superiores (região anterior) e distância intermolares superiores (região posterior).

Para a avaliação do erro intraexaminador, sete modelos foram aleatoriamente selecionados, sendo as medidas de interesse registradas em duas ocasiões, com intervalo de sete dias. Para a avaliação da concordância entre as medições, foi calculado o Coeficiente de Correlação Intraclasse (ICC), verificando-se a existência de correlação significativa entre as medições realizadas.

Para a comparação entre os momentos distintos (T1 e T2), foi utilizado o teste $t$ de Student para amostras pareadas. A comparação dos resultados obtidos entre os grupos foi realizada por meio da aplicação do teste de comparações de médias $t$ de Student para amostras independentes. Os resultados foram considerados significativos a um nível de significância máximo de $1 \%$.

\section{RESULTADOS}

A partir dos resultados obtidos, verificou-se que houve diferença significativa tanto na distância interpré-molares como na distância intermolares para os valores encontrados nos tempos $\mathrm{T} 1$ e T2, observando-se valores superiores no tempo T2 (Gráf. 1). Isso significa que houve um ganho transversal no 


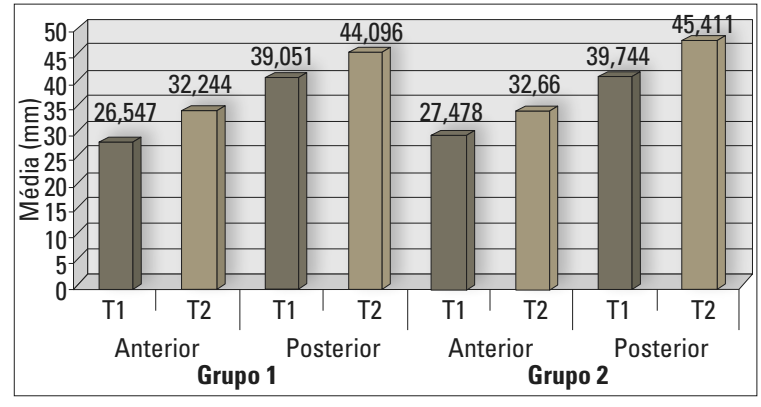

GRÁFICO 1 - Comparação entre os tempos T1 e T2 e entre as respectivas regiões do arco superior (região anterior = distância interpré-molares superiores, região posterior = distância intermolares superiores).

arco superior relacionado às regiões anterior e posterior do mesmo, atingindo, portanto, os objetivos do tratamento estabelecido.

Os dois protocolos de ativação mostraram-se igualmente efetivos na expansão maxilar, já que não houve diferença significativa entre os grupos quando comparadas as diferenças médias (T2 $\mathrm{T} 1$ ), portanto, o aumento transversal obtido foi o mesmo nos Grupos 1 e 2 (Tab. 1).

\section{DISCUSSÃO}

Os pacientes portadores de fissura apresentam não só prejuízos estéticos, mas principalmente funcionais. As alterações morfológicas das estruturas anatômicas diretamente atingidas provocam modificações na fala, alimentação e respiração, refletindo sobre o aspecto psicológico e dificultando a inserção desses indivíduos na sociedade ${ }^{1,6}$. Cabe ao ortodontista estabelecer uma oclusão mais próxima da ideal, por meio da conformação adequada dos arcos dentários, contribuindo da melhor maneira possível com esse tratamento que integra vários setores da Saúde.

A forma da maxila em portadores de fissura transforame incisivo é, em muitos casos, distorcida pela fenda. Para o estudo das modificações dos arcos maxilares, vários autores propuseram métodos para analisar a forma desses arcos em tais pacientes, sendo os principais os métodos descritivo e quantitativo.
TABELA 1 - Comparação das diferenças (T2-T1) entre os grupos.

\begin{tabular}{cccccc} 
grupo & n $\begin{array}{c}\text { diferença média } \\
\text { (T2-T1) }\end{array}$ & d.p. & t & Anterior \\
Grupo 1 & 7 & 5,697 & 2,020 & \\
Grupo 2 & 8 & 5,182 & 1,593 & 0,552 & 0,591 \\
& \multicolumn{5}{c}{ Posterior } \\
Grupo 1 & 7 & 5,045 & 1,549 & \\
Grupo 2 & 8 & 5,677 & 1,477 & $-0,797$ & 0,440
\end{tabular}

O método quantitativo consiste na determinação de três pares de pontos nos modelos, sendo um anterior, um mediano e outro posterior, correspondendo às distâncias intercaninos, interprémolares e intermolares, respectivamente - a análise dos valores dessas distâncias permite avaliar a forma do arco superior, bem como diagnosticar as deformidades ${ }^{5}$. Devido à irregularidade, na amostra utilizada, da posição dos caninos superiores, o que traria resultados não-fidedignos, decidiuse por utilizar apenas duas medidas transversais, sendo a interpré-molares denominada anterior em vista da ausência da medição intercaninos. Sto$\mathrm{ckli}^{25}$ apresentou um método onde há uma combinação de medidas transversais (larguras anterior, média e posterior do arco maxilar, anterior e posterior da fenda) e anteroposteriores (comprimento sagital do arco maxilar e dos segmentos maior e menor), com descrição da configuração da fenda alveolar, que pode ser neutra, positiva ou negativa.

A avaliação da discrepância transversal da maxila de indivíduos fissurados tem sido realizada, em muitos estudos, usando como referência pontos situados nos dentes superiores, como o ápice das cúspides dos caninos, pré-molares e molares. Santos $^{22}$ analisou a morfologia maxilar em fissurados transforame incisivo unilateral em modelos de gesso do arco dentário superior de 30 pacientes. Concluiu que, após a cirurgia de lábio, a maxila sofre uma constrição decrescente no sentido 
anteroposterior e um estreitamento da fenda. Logo após a cirurgia de palato, observou uma constrição lateral associada à ausência de crescimento anteroposterior significativo, a ponto de caracterizar uma fase de estagnação. A avaliação em longo prazo dos casos evidencia uma melhora no diâmetro transversal da maxila e a saída da fase de estagnação, com significativo crescimento anteroposterior.

Heidbuchel e Kuijpers-Jagtman ${ }^{10}$ examinaram 149 modelos de 22 pacientes, dos 3 aos 17 anos de idade, portadores de fissura transforame incisivo bilateral, operados, comparando-os com uma amostra de 42 crianças não-portadoras de fissura. O objetivo era avaliar a oclusão (severidade e localização de mordidas cruzadas) e as dimensões, em largura e profundidade, da maxila e da mandíbula, a partir de pontos demarcados nas estruturas dentárias. O grupo de fissurados apresentou um comprimento maxilar muito menor a partir dos 9 anos de idade, assim como também um arco dentário significativamente mais estreito durante todo o período estudado, com tendência à oclusão de topo, especialmente na região dos caninos.

Após as cirurgias de lábio e palato, a maxila sofre alterações na sua forma. Dentre as sequelas maxilares apresentadas pelos fissurados, as alterações de diâmetro transverso do arco superior são as mais frequentes ${ }^{5,17}$ e, se não tratadas, levam a deformidades faciais graves na idade adulta. A distância transversal anterior é a que sofre maior alteração, seguida da região mediana e depois da posterior $^{1}$. A expansão ortopédica é frequentemente requerida no tratamento de portadores de fissuras de lábio e palato ${ }^{16}$.

Em muitos casos, apenas a expansão maxilar não é suficiente para a obtenção de uma oclusão adequada nesses pacientes, sendo necessária sua associação com a protração da maxila. Isso se justifica quando há combinação de retrusão da face média, ângulo nasolabial agudo e excesso relativo da altura facial inferior, o que contribui para uma aparência desagradável nos pacientes fissurados, tendo grande influência em seu comportamento social, já que há alteração no perfil mole do terço facial inferior, envolvendo nariz, lábios e queixo ${ }^{26}$.

Para a expansão rápida da maxila (ERM) o protocolo de ativações estabelecido por $\mathrm{Haas}^{9}$ é muito difundido, obtendo resultados bastante satisfatórios. Em 2005, Liou e Tsai ${ }^{15}$ publicaram um novo protocolo de ativações, com expansões e contrações da maxila alternadas semanalmente. Esse protocolo é aplicado por sete a nove semanas para desarticular as suturas circum-maxilares sem sobre-expandir a maxila. Assim, na primeira semana o parafuso é expandido, na segunda semana contraído, na terceira expandido, e assim sucessivamente até a sétima ou nona semana. Ao final do protocolo, o expansor deve estar sempre aberto. Os autores utilizaram um disjuntor com duas dobradiças, ativando uma volta completa por dia, associado à protração maxilar.

Para avaliar os efeitos da protração maxilar com máscara facial em pacientes portadores de fissura transforame incisivo unilateral, Vieira ${ }^{29}$ comparou dois grupos, também de pacientes fissurados, que foram submetidos a dois protocolos distintos de ERM, sendo um o convencional e o outro o proposto por Liou e Tsai ${ }^{15}$. Não foram encontradas diferenças significativas em relação à quantidade de avanço da maxila. Nesse caso, o aparelho expansor utilizado foi o disjuntor de Haas modificado. Esse aparelho, com parafuso fixo ao palato e extensões em acrílico, maximiza o efeito esquelético durante a ERM, já que permite, durante sua ativação, a aplicação de forças mais pesadas sobre a maxila ${ }^{9,20}$.

No presente estudo foram analisadas quantitativamente as dimensões transversais do arco superior das amostras obtidas no trabalho de Vieira ${ }^{29}$, comparando-se, quanto à discrepância transversal, os dois protocolos de ativação durante a expansão maxilar, utilizando pontos situados nas regiões anterior e posterior do arco superior de pacientes portadores de fissura.

Os pontos utilizados foram dentários ${ }^{3,4,10}$, sendo as distâncias registradas, com um paquímetro 
digital de alta precisão, por um único operador. A distância interpré-molares, avaliada nos primeiros pré-molares superiores, representou a largura da região anterior do arco. A distância intermolares, medida nos primeiros molares superiores permanentes, determinou a largura da região posterior.

Apesar de ambos os grupos terem sido submetidos à mesma quantidade de abertura do parafuso expansor $(5,6 \mathrm{~mm})$, procurou-se, com essa pesquisa, investigar a presença de possíveis diferenças no comportamento da resposta frente às resistências teciduais à abertura do parafuso e às alterações morfológicas maxilares e faciais características dos pacientes portadores de fissuras. A dúvida inicial referia-se à influência das expansões e contrações alternadas (Grupo 2) - o que significou a atuação do aparelho por um período maior nas estruturas anatômicas - sobre o grau de abertura conseguido e se esse seria semelhante àquele obtido com apenas uma semana de manipulação do parafuso (Grupo 1). A resposta à expansão rápida da maxila em pacientes com fissura por meio de protocolos diferentes de ativação é pouco pesquisada, sendo de grande importância a realização de estudos que abordem esse assunto utilizando métodos cientificamente embasados.

Alguns estudos comparam não só diferentes protocolos de expansão, mas também sua realização com expansores de configurações distintas. Mossaz-Joëlson e Mossaz ${ }^{19}$ analisaram os efeitos da expansão lenta da maxila com dois aparelhos, sendo um bandado e o outro colado, não encontrando diferenças em relação à expansão esquelética e dentária obtida. Oliveira et $a 1 .^{20}$ realizaram um estudo tridimensional em modelos de gesso com o objetivo de avaliar as alterações na conformação do palato com o uso de dois aparelhos de expansão rápida da maxila (Haas e Hyrax). Os autores concluíram que ambos os dispositivos utilizados promoveram aumento da largura maxilar e da distância intermolares, sendo que o aparelho tipo Haas promoveu um movimento ortopédico superior.

Akkaya, Lorenzon e Üçem ${ }^{3}$ compararam, em modelos ortodônticos, os efeitos dentários obtidos com o uso de dois tipos de expansores fixos, sendo em um deles realizada a expansão rápida e no outro a lenta. Cada grupo era constituído por 12 pacientes com média de idades de 12 anos, mordida cruzada posterior bilateral e não-portadores de fissura. Foram registradas as distâncias transversais da maxila correspondentes aos caninos, primeiros pré-molares e molares superiores, sendo encontrado um ganho significativamente maior na distância intercaninos superiores produzido pela expansão rápida.

No presente estudo, quando comparadas as medidas transversais iniciais e finais, todas apresentaram aumento significativo, o que demonstra a efetividade dos dois protocolos de expansão instituídos. Contudo, ao se efetuar a comparação intergrupos quanto ao ganho transversal, não se encontrou diferença estatisticamente significativa. Vieira ${ }^{29}$ também não encontrou diferenças significativas quanto ao avanço da maxila nessa mesma amostra.

Liou e Tsai ${ }^{15}$ não avaliaram os parâmetros utilizados nesse estudo, não sendo, portanto, possível a comparação com o seu trabalho. Entretanto, pode-se esperar alguma diferença atribuída ao tipo de expansor utilizado pelos autores. Dessa forma, trabalhos que utilizem a mesma metodologia são necessários.

\section{CONCLUSÃO}

Com base na análise dos resultados obtidos, conclui-se que os dois protocolos de ativação foram igualmente efetivos na expansão maxilar, não havendo diferença em relação ao aumento transversal obtido. Sendo assim, ambos podem ser utilizados quando o intuito do tratamento é obter um aumento no diâmetro transversal do arco superior.

Enviado em: novembro de 2007 Revisado e aceito: outubro de 2008 


\title{
Morphological analysis of the maxillary arch in patients with cleft lip and palate submitted to different protocols of rapid maxillary expansion: Maxillary altera- tions evaluation
}

\begin{abstract}
Introduction: Harmonious conformation of the maxillary arch in patients with cleft lip and palate is one of the main requirements for achievement of adequate occlusion and for esthetic and functional improvement. For this purpose, orthopedic maxillary expansion is often required due to the high frequency of the segments collapse. Aim: This study aimed to evaluate the transverse dimensions of maxillary dental arches by analysis of the dental casts of 15 patients with complete unilateral cleft lip and palate who presented sagittal and transverse maxillary constriction and were submitted to two distinct protocols of rapid maxillary expansion. Methods: Seven patients were submitted to rapid maxillary expansion (RME) by the conventional activation protocol (Group 1), and the others were treated by the protocol proposed by Liou and Tsai in 2005, with alternated expansion and constriction (Group 2). Both groups were later submitted to maxillary protraction with facial mask. Dental casts were obtained at two periods: before placement of a modified Haas expander (T1) and after 24 weeks of treatment (T2). Transverse distances were measured with a digital caliper to register the distances between first upper premolars (anterior area) and first upper molars (posterior area). Data were statistically analyzed by the Student's t test. Results and

Conclusion: Both protocols were equally effective, since no differences were observed between the groups as to the transverse increasing achieved.
\end{abstract}

Keywords: Maxillary expansion. Interbicuspids width. Intermolars width. Cleft lip and palate.

\section{REFERÊNCIAS}

1. $A B D O$, R. C. C. Análise morfológica do arco maxilar superior em portadores de fissura transforame incisivo unilateral, operados: estudo longitudinal (3 aos 9 anos de idade). 1988. Tese (Livre Docência em Odontopediatria)-Faculdade de Odontologia de Bauru, Universidade de São Paulo, Bauru, 1988.

2. ABDO, R. C. C.; MACHADO, M. A. A. M. Odontopediatria nas fissuras lábio-palatais. São Paulo: Ed. Santos, 2005.

3. AKKAYA, S.; LORENZON, S.; ÜÇEM, T. T. Comparison of dental arch and arch perimeter changes between bonded rapid and slow maxillary expansion procedures. Eur. J. Orthod., Oxford, v. 20, no. 2, p. 255-261, June 1988.

4. ATHANASIOU, A. E. et al. Dental arch dimensions in patients with unilateral cleft lip and palate. Cleft Palate J., Pittsburgh, v. 25 , no. 2 , p. 139-145, Apr. 1988.

5. CAPELOZZA FILHO, L. Análise do diâmetro transverso dos maxilares em portadores de fissura transforame incisivo unilateral. 1976. Dissertação (Mestrado)-Faculdade de Odontologia de Bauru, Universidade de São Paulo, Bauru, 1976.

6. CAPELOZZA FILHO, L.; MARTINS, D. R.; MAZZOTTINI, R. Análise do diâmetro transverso do maxilar superior de portadores de fissura transforame incisivo unilateral. Ars. Curandi Odont. São Paulo, v. 6, n. 6, p. 42-51, set. 1979.

7. CAPELOZZA FILHO, L.; MAZZOTTINI, R.; ABDO, R. C. C. Expansão rápida da maxila em fissurados adultos. Ars. Curandi Odont., São Paulo, v. 7, n. 5, p. 209-224, ago. 1979.
8. GOTTLIEB, M. A. Defectos congénitos. In: NOWAK, A. J. Odontologia para el paciente impedido. Argentina: Mundi, 1979. p. 58-69.

9. HAAS, A. J. Rapid expansion of the maxillary dental arch and nasal cavity by opening the midpalatal suture. Angle Orthod., Appleton, v. 31, no. 2, p. 37-90, Apr. 1961.

10. HEIDBUCHEL, K. L.; KUIJPERS-JAGTMAN, A. M. Maxillary and mandibular dental-arch dimensions and occlusion in bilateral cleft lip and palate patients from 3 to 17 years of age. Cleft Palate Craniofac. J., Pittsburgh, v. 34, no. 1, p. 21-26, Jan. 1997.

11. ISAACSON, R. J.; MURPHY, T. D. Some effects of rapid palatal expansion in cleft lip and palate patients. Angle Orthod., Appleton, v. 34, no. 3, p. 143-154, July 1964.

12. ISHIKAWA, H.; KITAZAWA, S.; IWASAKI, H.; NAKAMURA, S. Effects of maxillary protraction combined with chin-cap therapy in unilateral cleft lip and palate patients. Cleft Palate Craniofac. J., Pittsburgh, v. 37, no. 1, p. 92-97, Jan. 2000.

13. LANES, M. A. Expansão rápida maxilar com parafuso convencional e limitador posterior em pacientes com fissura transforame incisivo: alterações verticais. 2006. $145 \mathrm{f}$. Dissertação (Mestrado)-Pontifícia Universidade Católica do Rio Grande do Sul, Porto Alegre, 2006.

14. LAPA, F. S.; SPINA, V. Ortodontia aplicada nas fissuras lábiopalatinas. Rev. Hosp. Clín. Fac. Méd. S. Paulo, São Paulo, v. 16, n. 3, p. 208-214, maio/jun. 1961. 
15. LIOU, E. J.: TSAI, W. C. A new protocol for maxillary protraction in cleft patients: Repetitive weekly protocol of alternate rapid maxillary expansions and constrictions. Cleft Palate Craniofac. J., Pittsburgh, v. 42, no. 2, p. 121-127, Mar. 2005

16. LONG JR., R. E.; SEMB, G. S.; SHAW, W. C. Orthodontic treatment of the patient with complete lip and palate: Lessons of the past 60 years. Cleft Palate Craniofac. J., Pittsburgh, v. 37 no. 6, p. 533-531, 533-413, Nov. 2000.

17. MARCUSSON, A.; PAULIN, G. Changes in occlusion and maxillary dental arch dimensions in adults with treated unilateral complete cleft lip and palate: A follow-up study. Eur. J. Orthod., Oxford, v. 26, no. 4, p. 385-390, Apr. 2004

18. MARS, M. et al. A six-center international study of treatment outcome in patients with cleft of the lip and palate. Part 3: Dental arch relationship. Cleft Palate Craniofac. J., Pittsburgh, v. 29 , no. 5 , p. 405-408, Sept. 1992.

19. MOSSAZ-JOËLSON, K.; MOSSAZ, C. F. Slow maxillary expansion: A comparison between banded and bonded appliances. Eur. J. Orthod., Oxford, v. 11, no. 1, p. 67-76, Feb. 1989.

20. OLIVEIRA, N. L.; SILVEIRA, A. C.; KUSNOTO, B.; VIANA, C. Three-dimensional assessment of morphologic changes of the maxilla: A comparison of 2 kinds of palatal expanders. Am. J. Orthod. Dentofacial Orthop., St. Louis, v. 126, no. 3, p. 354-362, Sept. 2004.

21. ROSS, R. B. The clinical implications of facial growth in cleft lip and palate. Cleft Palate J., Pittsburgh, v. 7, no. 1, p. 37-47, Jan. 1970.

22. SANTOS, S. D. Avaliação morfológica do arco dentário maxilar de portadores de fissuras transforame incisivo unilateral. 1986. Trabalho de Conclusão de Curso (Especialização)Hospital de Reabilitação de Lesões Lábio-Palatais da Universidade de São Paulo, Bauru, 1986.
23. SILVA FILHO, O. G.; CAPELOZZA FILHO, L.; WERNECK, V. A . FREITAS, J. A. S. Abordagem ortodôntica ao paciente com fissura unilateral completa de lábio e palato. Rev. Soc. Paul. Ortod., São Paulo, v. 31, n. 3, p. 32-44, set./dez. 1998

24. SO, L. L. Effects of reverse headgear treatment on sagittal correction in girls born with unilateral complete cleft lip and cleft palate - skeletal and dental changes. Am. J. Orthod. Dentofacial Orthop., St. Louis, v. 109, no. 2, p. 140-147, Feb. 1970.

25. STOCKLI, P. W. Application of a quantitative method for arch form evaluation in complete unilateral cleft lip and palate.

Cleft Palate J., Pittsburgh, v. 8, no. 4, p. 322-341, July 1971

26. TINDLUND, R. S.; RYGH, P. Soft tissue profile changes during widening and protraction of the maxilla in patients with cleft lip and palate compared with normal growth and development. Cleft Palate Craniofac. J., Pittsburgh, v. 30, no. 5, p. 454-468, Sept. 1993.

27. TINDLUND, R. S.; RYGH, P.; BOE, O. E. Intercanine widening and sagittal effect of maxillary transverse expansion in patients with cleft lip and palate during the deciduous and mixed dentitions. Cleft Palate Craniofac. J., Pittsburgh, v. 30, no. 2 p. 195-207, Mar. 1993

28. TURLEY, R. Orthopedic correction of Class III malocclusion with palatal expansion and custom protraction headgear. J. Clin. Orthod., Boulder, v. 22, no. 5, p. 314-325, May 1988.

29. VIEIRA, G. L. Comparação da protração maxilar em pacientes portadores de fissura lábio-palatal por meio de dois protocolos de expansão rápida maxilar. 2006. $157 \mathrm{f}$. Dissertação (Mestrado)-Pontifícia Universidade Católica do Rio Grande do Sul, Porto Alegre, 2006.
Endereço para correspondência

Faculdade de Odontologia / PUC-RS - Departamento de Odontologia Preventiva, Disciplina de Ortodontia

Maíra Massuia de Souza

Av. Ipiranga, 6681 - prédio 06

CEP: $90.619-900$ - Porto Alegre/ RS - Caixa Postal 1429

E-mail: mairamassuia@gmail.com 